\title{
Revisiting the hexagonal lattice: on optimal lattice circle packing
}

\author{
Lenny Fukshansky \\ Lenny Fukshansky received his Ph.D. from the University of Texas at Austin in 2004 \\ Since 2007 he holds a position at Claremont McKenna College in California (USA), \\ where he is now an associate professor in the Department of Mathematics. His main \\ fields of research are number theory, diophantine geometry, and discrete geometry.
}

\section{Introduction}

The classical circle packing problem asks for an arrangement of nonoverlapping circles in $\mathbb{R}^{2}$ so that the largest possible proportion of the space is covered by them. This problem has a long and fascinating history with its origins in the works of Albrecht Dürer and Johannes Kepler. The answer to this is now known: the largest proportion of the real plane, about $90.7 \%$, is covered by the arrangement of circles with centers at the points of the hexagonal lattice. The first claim of a proof was made by Axel Thue in 1892, and then once again in 1910. It is generally believed however that the first complete flawless proof was produced only in 1940 by László Fejes-Tóth (see [2], [10] for detailed accounts and bibliography). On the other hand, the fact that the hexagonal lattice gives the maximal possible circle packing density among all lattice arrangements has been known

Die Aufgabe, die dichteste Packung der Ebene bzw. des Raumes mit nicht überlappenden, kongruenten Kreisen bzw. Kugeln zu finden, ist ein klassisches Problem, mit dem sich bereits Johannes Kepler befasst hat. Im Falle der Ebene liegt seit 1940 durch László Fejes-Tóth ein vollständiger Beweis der Tatsache vor, dass die dichteste Kreispackung der Ebene aus einem hexagonalen Gitter, d.h. aus einem Bienenwabenmuster, hervorgeht und damit ca. 90,7\% der Ebene überdeckt werden. In dem nachfolgenden Beitrag gibt der Autor einen elementaren Beweis des in diesem Kontext wichtigen Teilergebnisses, dass unter den Kreispackungen der Ebene, die aus Gittern hervorgehen, das hexagonale Gitter zur optimalen Lösung führt. Was die dichteste Kugelpackung des Raumes, die sogenannte Keplersche Vermutung, anbetrifft, so wurde diese im Jahr 1998 durch Thomas C. Hales unter Verwendung des Computers bewiesen. 
much earlier: all the necessary ingredients for the first such proof were present already in the work of Lagrange, although he himself, while aware of the circle packing problem, may not have realized that he essentially had a proof for the optimal lattice packing in hands. In fact, the notion of a lattice has not been formally introduced until the work of Gauss in 1831. A detailed history and overview of these and other developments in the direction of the circle packing problem and its (much more difficult) three-dimensional analogue, the Kepler conjecture, can be found in the excellent recent book of G.G. Szpiro [12].

In this note we concentrate on the lattice circle packing problem. Let us first set up the basic notation and describe the problem. Recall that a lattice $\Lambda$ in $\mathbb{R}^{2}$ is a free $\mathbb{Z}$-module of rank two, so $\Lambda=X \mathbb{Z}^{2}$ for some matrix $X=\left(\boldsymbol{x}_{1} \boldsymbol{x}_{2}\right) \in \mathrm{GL}_{2}(\mathbb{R})$, where the column vectors $\boldsymbol{x}_{1}, \boldsymbol{x}_{2}$ of $X$ form a basis for $\Lambda$ and $X$ is referred to as the corresponding basis matrix. The determinant of $\Lambda$, denoted by $\operatorname{det}(\Lambda)$, is defined to be $|\operatorname{det}(X)|$, which does not depend on the particular choice of a basis for $\Lambda$. Let us now construct a circle packing associated to $\Lambda$. Define the Voronoi cell of $\Lambda$ to be

$$
\mathcal{V}(\Lambda)=\left\{\boldsymbol{y} \in \mathbb{R}^{2}:\|\boldsymbol{y}\| \leq\|\boldsymbol{y}-\boldsymbol{x}\| \forall \boldsymbol{x} \in \Lambda\right\},
$$

where we write \|\| for the Euclidean norm on $\mathbb{R}^{2}$. In other words, $\mathcal{V}(\Lambda)$ is the closure of the set of all vectors in the real plane which are closer to $\mathbf{0}$ than to any other vector of $\Lambda$. The area of the Voronoi cell is equal to $\operatorname{det}(\Lambda)$, and

$$
\mathbb{R}^{2}=\bigcup_{\boldsymbol{y} \in \Lambda} \mathcal{V}(\Lambda)+\boldsymbol{y}
$$

meaning that the real plane is tiled with the translates of $\mathcal{V}(\Lambda)$. Moreover, as is clear from the definition, the interiors of these translates are disjoint. Let us inscribe a circle into each translate $\mathcal{V}(\Lambda)+\boldsymbol{y}$ of this Voronoi cell by a point of the lattice, and write $r(\Lambda)$ for the radius of this circle. No two such circles overlap, and so we have a circle packing in $\mathbb{R}^{2}$, called the lattice packing corresponding to $\Lambda$. The density of this circle packing is now given by

$$
\Delta(\Lambda)=\frac{\text { area of one circle }}{\text { area of the Voronoi cell }}=\frac{\pi r(\Lambda)^{2}}{\operatorname{det}(\Lambda)} .
$$

The lattice packing problem in $\mathbb{R}^{2}$ is to maximize this density function on the space of all lattices. The answer has been known since the end of the nineteenth century (see Fig. 1): this density function $\Delta$ on lattices in $\mathbb{R}^{2}$ is maximized by the hexagonal lattice

$$
\Lambda_{h}:=\left(\begin{array}{cc}
1 & \frac{1}{2} \\
0 & \frac{\sqrt{3}}{2}
\end{array}\right) \mathbb{Z}^{2} .
$$

Here we will present a proof of this fact, emphasizing the particular properties of $\Lambda_{h}$ that make it a solution to this optimization problem.

Let us say that two lattices $\Lambda$ and $\Omega$ in $\mathbb{R}^{2}$ are similar if there exists a real constant $\alpha$ and a $2 \times 2$ orthogonal real matrix $U$ such that

$$
\Omega=\alpha U \Lambda,
$$




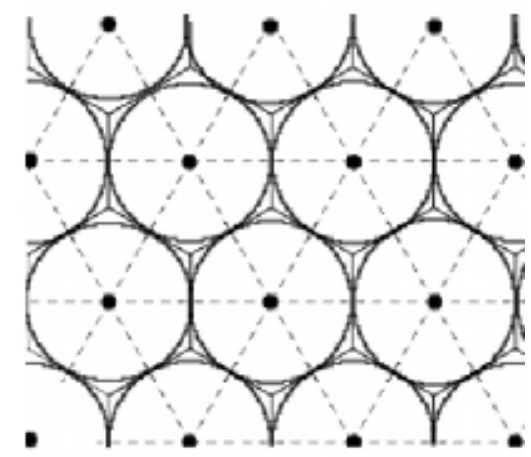

Fig. 1 Hexagonal lattice with Voronoi cell translates and associated circle packing

in other words, if $\Omega$ can be obtained from $\Lambda$ by rotation and dilation. Similarity is readily seen to be an equivalence relation, and it is easy to notice that the packing density function $\Delta$ is constant on each similarity class. We will prove the following classical result.

Theorem 1.1. Let $\Lambda$ be a lattice of rank 2 in $\mathbb{R}^{2}$. Then

$$
\Delta(\Lambda) \leq \Delta\left(\Lambda_{h}\right)=\frac{\pi}{2 \sqrt{3}}=0.906899 \ldots
$$

with equality in (1) if and only if $\Lambda$ is similar to $\Lambda_{h}$.

\section{Background and standard notation}

We start by setting up some additional notation. Let $B$ be the unit circle centered at the origin in $\mathbb{R}^{2}$. Given a lattice $\Lambda$, we define Minkowski successive minima $\lambda_{1} \leq \lambda_{2}$ of $\Lambda$ to be

$$
\lambda_{i}=\inf \left\{\lambda \in \mathbb{R}_{>0}: \Lambda \cap \lambda B \text { contains } i \text { linearly independent nonzero vectors }\right\},
$$

where $i=1,2$. By definition of the Voronoi cell of $\Lambda$, its in-radius is equal to one half of the distance from the origin to the nearest (with respect to Euclidean norm) point of $\Lambda$, which is precisely $\lambda_{1} / 2$, and so

$$
\Delta(\Lambda)=\frac{\pi \lambda_{1}^{2}}{4 \operatorname{det}(\Lambda)} .
$$

We will say that the vectors $\boldsymbol{x}_{1}, \boldsymbol{x}_{2} \in \Lambda$ correspond to successive minima $\lambda_{1}, \lambda_{2}$ if they are linearly independent and

$$
\left\|x_{1}\right\|=\lambda_{1},\left\|x_{2}\right\|=\lambda_{2} .
$$

Notice that if $\boldsymbol{x}_{1}, \boldsymbol{x}_{2}$ correspond to successive minima in $\Lambda$, then so do $\pm \boldsymbol{x}_{1}, \pm \boldsymbol{x}_{2}$. From now on, when we refer to vectors corresponding to successive minima in a lattice in $\mathbb{R}^{2}$, 
we will always mean a pair of such vectors so that the angle $\theta$ between them is in the interval $[0, \pi / 2]$. Therefore $\cos \theta>0$, and so

$$
\boldsymbol{x}_{1}^{t} \boldsymbol{x}_{2}=\left\|\boldsymbol{x}_{1}\right\|\left\|\boldsymbol{x}_{2}\right\| \cos \theta>0 .
$$

A lattice $\Lambda \subset \mathbb{R}^{2}$ is called well-rounded, abbreviated WR, if its successive minima $\lambda_{1}$ and $\lambda_{2}$ are equal. The hexagonal lattice $\Lambda_{h}$ is an example of a WR lattice with $\lambda_{1}=\lambda_{2}=1$. Well-rounded lattices are very important in coding theory [1] and discrete optimization problems [7]; they also come up in the context of some number theoretic problems, such as Minkowski's conjecture [8] and the linear Diophantine problem of Frobenius [5]. For a detailed study of the distribution of certain types of WR lattices in $\mathbb{R}^{2}$ see [3] and [4]. In Lemma 3.7 below we show that the WR property is preserved under similarity, i.e. a well-rounded lattice in $\mathbb{R}^{2}$ can only be similar to another well-rounded lattice, and give a simple necessary and sufficient criterion for two WR lattices in $\mathbb{R}^{2}$ to be similar. Thus Theorem 1.1 implies right away that only a WR lattice can maximize lattice packing density.

Our proof of Theorem 1.1 emphasizes the importance of WR lattices. Specifically, we first prove that $\Delta$ must achieve its maximum at a WR lattice, hence this optimization problem can be restricted to WR lattices only. Next we show that if $\Lambda$ is WR, then $\Delta(\Lambda)$ is given by a particularly simple expression, and maximizing it becomes an easy problem. Our argument is self-contained and requires no background beyond linear algebra. For further topics in the fascinating subject of lattice packing in dimensions two and higher see [2], [6], [7], [10], and [11]. We are now ready to proceed.

\section{Properties of well-rounded lattices in $\mathbb{R}^{2}$}

Our goal here is to prove that the circle packing density function on the space of all lattices in $\mathbb{R}^{2}$ achieves its maximum at the hexagonal lattice. We start with a simple, but very useful lemma.

Lemma 3.1. Let $\boldsymbol{x}_{1}$ and $\boldsymbol{x}_{2}$ be nonzero vectors in $\mathbb{R}^{2}$ so that the angle $\theta$ between them satisfies $0<\theta<\pi / 3$. Then

$$
\left\|\boldsymbol{x}_{1}-\boldsymbol{x}_{2}\right\|<\max \left\{\left\|\boldsymbol{x}_{1}\right\|,\left\|\boldsymbol{x}_{2}\right\|\right\} .
$$

Proof. Notice that $\boldsymbol{x}_{1}^{t} \boldsymbol{x}_{2}>0$ by (3). Then, since $\theta<\pi / 3$,

$$
\frac{1}{2}<\cos \theta=\frac{\boldsymbol{x}_{1}^{t} \boldsymbol{x}_{2}}{\left\|\boldsymbol{x}_{1}\right\|\left\|\boldsymbol{x}_{2}\right\|},
$$

and hence

$$
\begin{aligned}
\left\|x_{1}-x_{2}\right\|^{2} & =\left(x_{1}-x_{2}\right)^{t}\left(x_{1}-x_{2}\right)=\left\|x_{1}\right\|^{2}+\left\|x_{2}\right\|^{2}-2 x_{1}^{t} x_{2} \\
& <\left\|x_{1}\right\|^{2}+\left\|x_{2}\right\|^{2}-\left\|x_{1}\right\|\left\|x_{2}\right\|<\max \left\{\left\|x_{1}\right\|,\left\|x_{2}\right\|\right\}^{2} .
\end{aligned}
$$

Lemma 3.1 readily implies that the angle between vectors corresponding to successive minima in a lattice cannot be $<\pi / 3$. 
Lemma 3.2. Let $\Lambda \subset \mathbb{R}^{2}$ be a lattice of full rank with successive minima $\lambda_{1} \leq \lambda_{2}$, and let $\boldsymbol{x}_{1}, \boldsymbol{x}_{2}$ be the vectors in $\Lambda$ corresponding to $\lambda_{1}, \lambda_{2}$, respectively. Let $\theta \in[0, \pi / 2]$ be the angle between $\boldsymbol{x}_{1}$ and $\boldsymbol{x}_{2}$. Then

$$
\pi / 3 \leq \theta \leq \pi / 2
$$

Proof. Assume that $\theta<\pi / 3$, then Lemma 3.1 implies that

$$
\left\|x_{1}-x_{2}\right\|<\left\|x_{2}\right\|=\lambda_{2}
$$

which contradicts the definition of $\lambda_{2}$ since the vectors $\boldsymbol{x}_{1}$ and $\boldsymbol{x}_{1}-\boldsymbol{x}_{2}$ are linearly independent.

We can now prove that vectors corresponding to successive minima in a lattice in $\mathbb{R}^{2}$ form a basis.

Lemma 3.3. Let $\Lambda$ be a lattice in $\mathbb{R}^{2}$ with successive minima $\lambda_{1} \leq \lambda_{2}$ and let $\boldsymbol{x}_{1}, \boldsymbol{x}_{2}$ be the vectors in $\Lambda$ corresponding to $\lambda_{1}, \lambda_{2}$, respectively. Then $\boldsymbol{x}_{1}, \boldsymbol{x}_{2}$ form a basis for $\Lambda$.

Proof. Let $\boldsymbol{y}_{1} \in \Lambda$ be a shortest vector extendable to a basis in $\Lambda$, and let $\boldsymbol{y}_{2} \in \Lambda$ be a shortest vector such that $\boldsymbol{y}_{1}, \boldsymbol{y}_{2}$ is a basis of $\Lambda$. By picking $\pm \boldsymbol{y}_{1}, \pm \boldsymbol{y}_{2}$ if necessary we can ensure that the angle between these vectors is no greater than $\pi / 2$. Then

$$
0<\left\|\boldsymbol{y}_{1}\right\| \leq\left\|\boldsymbol{y}_{2}\right\|
$$

and for any vector $z \in \Lambda$ with $\|\boldsymbol{z}\|<\left\|\boldsymbol{y}_{2}\right\|$ the pair $\boldsymbol{y}_{1}, \boldsymbol{z}$ is not a basis for $\Lambda$. Since $\boldsymbol{x}_{1}, \boldsymbol{x}_{2} \in \Lambda$, there must exist integers $a_{1}, a_{2}, b_{1}, b_{2}$ such that

$$
\left(\begin{array}{ll}
\boldsymbol{x}_{1} & \boldsymbol{x}_{2}
\end{array}\right)=\left(\begin{array}{ll}
\boldsymbol{y}_{1} & \boldsymbol{y}_{2}
\end{array}\right)\left(\begin{array}{ll}
a_{1} & b_{1} \\
a_{2} & b_{2}
\end{array}\right)
$$

Let $\theta_{x}$ be the angle between $\boldsymbol{x}_{1}, \boldsymbol{x}_{2}$, and $\theta_{y}$ be the angle between $\boldsymbol{y}_{1}, \boldsymbol{y}_{2}$, then $\pi / 3 \leq \theta_{x} \leq$ $\pi / 2$ by Lemma 3.2. Moreover, $\pi / 3 \leq \theta_{y} \leq \pi / 2$ : indeed, suppose $\theta_{y}<\pi / 3$, then by Lemma 3.1,

$$
\left\|\boldsymbol{y}_{1}-\boldsymbol{y}_{2}\right\|<\left\|\boldsymbol{y}_{2}\right\|,
$$

however $\boldsymbol{y}_{1}, \boldsymbol{y}_{1}-\boldsymbol{y}_{2}$ is a basis for $\Lambda$ since $\boldsymbol{y}_{1}, \boldsymbol{y}_{2}$ is; this contradicts the choice of $\boldsymbol{y}_{2}$. Define

$$
\mathcal{D}=\left|\operatorname{det}\left(\begin{array}{ll}
a_{1} & b_{1} \\
a_{2} & b_{2}
\end{array}\right)\right|
$$

then $\mathcal{D}$ is a positive integer, and taking determinants of both sides of (4), we obtain

$$
\left\|\boldsymbol{x}_{1}\right\|\left\|\boldsymbol{x}_{2}\right\| \sin \theta_{x}=\mathcal{D}\left\|\boldsymbol{y}_{1}\right\|\left\|\boldsymbol{y}_{2}\right\| \sin \theta_{y} .
$$

Notice that by definition of successive minima, $\left\|\boldsymbol{x}_{1}\right\|\left\|\boldsymbol{x}_{2}\right\| \leq\left\|\boldsymbol{y}_{1}\right\|\left\|\boldsymbol{y}_{2}\right\|$, and hence (5) implies that

$$
\mathcal{D}=\frac{\left\|\boldsymbol{x}_{1}\right\|\left\|\boldsymbol{x}_{2}\right\|}{\left\|\boldsymbol{y}_{1}\right\|\left\|\boldsymbol{y}_{2}\right\|} \frac{\sin \theta_{x}}{\sin \theta_{y}} \leq \frac{2}{\sqrt{3}}<2,
$$


meaning that $\mathcal{D}=1$. Combining this observation with (4), we see that

$$
\left(\begin{array}{ll}
\boldsymbol{x}_{1} & \boldsymbol{x}_{2}
\end{array}\right)\left(\begin{array}{ll}
a_{1} & b_{1} \\
a_{2} & b_{2}
\end{array}\right)^{-1}=\left(\begin{array}{ll}
\boldsymbol{y}_{1} & \boldsymbol{y}_{2}
\end{array}\right)
$$

where the matrix $\left(\begin{array}{ll}a_{1} & b_{1} \\ a_{2} & b_{2}\end{array}\right)^{-1}$ has integer entries. Therefore $\boldsymbol{x}_{1}, \boldsymbol{x}_{2}$ is also a basis for $\Lambda$, completing the proof.

Remark. We note that if we replace $\mathbb{R}^{2}$ with $\mathbb{R}^{d}$ then the statement of Lemma 3.3 is no longer true for $d \geq 5$ (see for instance [9]).

We will call a basis for a lattice as in Lemma 3.3 a minimal basis. The goal of the next three lemmas is to show that the lattice packing density function $\Delta$ attains its maximum in $\mathbb{R}^{2}$ on the set of well-rounded lattices.

Lemma 3.4. Let $\Lambda$ and $\Omega$ be lattices of full rank in $\mathbb{R}^{2}$ with successive minima $\lambda_{1}(\Lambda), \lambda_{2}(\Lambda)$ and $\lambda_{1}(\Omega), \lambda_{2}(\Omega)$, respectively. Let $\boldsymbol{x}_{1}, \boldsymbol{x}_{2}$ and $\boldsymbol{y}_{1}, \boldsymbol{y}_{2}$ be vectors in $\Lambda$ and $\Omega$, respectively, corresponding to successive minima. Suppose that $\boldsymbol{x}_{1}=\boldsymbol{y}_{1}$, and angles between the vectors $\boldsymbol{x}_{1}, \boldsymbol{x}_{2}$ and $\boldsymbol{y}_{1}, \boldsymbol{y}_{2}$ are equal, call this common value $\theta$. Suppose also that

$$
\lambda_{1}(\Lambda)=\lambda_{2}(\Lambda)
$$

Then

$$
\Delta(\Lambda) \geq \Delta(\Omega)
$$

Proof. By Lemma 3.3, $\boldsymbol{x}_{1}, \boldsymbol{x}_{2}$ and $\boldsymbol{y}_{1}, \boldsymbol{y}_{2}$ are minimal bases for $\Lambda$ and $\Omega$, respectively. Notice that

$$
\begin{aligned}
\lambda_{1}(\Lambda) & =\lambda_{2}(\Lambda)=\left\|x_{1}\right\|=\left\|x_{2}\right\| \\
& =\left\|y_{1}\right\|=\lambda_{1}(\Omega) \leq\left\|y_{2}\right\|=\lambda_{2}(\Omega) .
\end{aligned}
$$

Then, by (2),

$$
\begin{aligned}
\Delta(\Lambda) & =\frac{\pi \lambda_{1}(\Lambda)^{2}}{4 \operatorname{det}(\Lambda)}=\frac{\pi \lambda_{1}(\Lambda)^{2}}{4\left\|\boldsymbol{x}_{1}\right\|\left\|\boldsymbol{x}_{2}\right\| \sin \theta}=\frac{\pi}{4 \sin \theta} \\
& \geq \frac{\pi \lambda_{1}(\Omega)^{2}}{4\left\|\boldsymbol{y}_{1}\right\|\left\|\boldsymbol{y}_{2}\right\| \sin \theta}=\frac{\pi \lambda_{1}(\Omega)^{2}}{4 \operatorname{det}(\Omega)}=\Delta(\Omega) .
\end{aligned}
$$

The following lemma is a converse to Lemma 3.2.

Lemma 3.5. Let $\Lambda \subset \mathbb{R}^{2}$ be a lattice of full rank, and let $\boldsymbol{x}_{1}, \boldsymbol{x}_{2}$ be a basis for $\Lambda$ such that

$$
\left\|x_{1}\right\|=\left\|x_{2}\right\|,
$$

and the angle $\theta$ between these vectors lies in the interval $[\pi / 3, \pi / 2]$. Then $\boldsymbol{x}_{1}, \boldsymbol{x}_{2}$ is a minimal basis for $\Lambda$. In particular, this implies that $\Lambda$ is WR. 
Proof. Let $z \in \Lambda$, then $z=a \boldsymbol{x}_{1}+b \boldsymbol{x}_{2}$ for some $a, b \in \mathbb{Z}$. Then

$$
\|z\|^{2}=a^{2}\left\|\boldsymbol{x}_{1}\right\|^{2}+b^{2}\left\|\boldsymbol{x}_{2}\right\|^{2}+2 a b \boldsymbol{x}_{1}^{t} \boldsymbol{x}_{2}=\left(a^{2}+b^{2}+2 a b \cos \theta\right)\left\|\boldsymbol{x}_{1}\right\|^{2} .
$$

If $a b \geq 0$, then clearly $\|z\|^{2} \geq\left\|x_{1}\right\|^{2}$. Now suppose $a b<0$, then again

$$
\|z\|^{2} \geq\left(a^{2}+b^{2}-|a b|\right)\left\|x_{1}\right\|^{2} \geq\left\|x_{1}\right\|^{2},
$$

since $\cos \theta \leq 1 / 2$. Therefore $\boldsymbol{x}_{1}, \boldsymbol{x}_{2}$ are shortest nonzero vectors in $\Lambda$, hence they correspond to successive minima, and so form a minimal basis. Thus $\Lambda$ is WR, and this completes the proof.

Lemma 3.6. Let $\Lambda$ be a lattice in $\mathbb{R}^{2}$ with successive minima $\lambda_{1}, \lambda_{2}$ and corresponding basis vectors $\boldsymbol{x}_{1}, \boldsymbol{x}_{2}$, respectively. Then the lattice

$$
\Lambda_{\mathrm{WR}}=\left(x_{1} \frac{\lambda_{1}}{\lambda_{2}} x_{2}\right) \mathbb{Z}^{2}
$$

is WR with successive minima equal to $\lambda_{1}$.

Proof. By Lemma 3.2, the angle $\theta$ between $\boldsymbol{x}_{1}$ and $\boldsymbol{x}_{2}$ is in the interval $[\pi / 3, \pi / 2]$, and clearly this is the same as the angle between the vectors $\boldsymbol{x}_{1}$ and $\frac{\lambda_{1}}{\lambda_{2}} \boldsymbol{x}_{2}$. Then by Lemma $3.5, \Lambda_{\mathrm{WR}}$ is WR with successive minima equal to $\lambda_{1}$.

Now combining Lemma 3.4 with Lemma 3.6 implies that

$$
\Delta\left(\Lambda_{\mathrm{WR}}\right) \geq \Delta(\Lambda)
$$

for any lattice $\Lambda \subset \mathbb{R}^{2}$, and (6) readily implies that the equality in (7) occurs if and only if $\Lambda=\Lambda_{\mathrm{WR}}$, which happens if and only if $\Lambda$ is well-rounded. Therefore the maximum packing density among lattices in $\mathbb{R}^{2}$ must occur at a WR lattice, and so for the rest of this section we talk about WR lattices only. Next observation is that for any WR lattice $\Lambda$ in $\mathbb{R}^{2}$, (6) implies:

$$
\sin \theta=\frac{\pi}{4 \Delta(\Lambda)},
$$

meaning that $\sin \theta$ is an invariant of $\Lambda$, and does not depend on the specific choice of the minimal basis. Since by our conventional choice of the minimal basis and Lemma 3.2, this angle $\theta$ is in the interval $[\pi / 3, \pi / 2]$, it is also an invariant of the lattice, and we call it the angle of $\Lambda$, denoted by $\theta(\Lambda)$.

Lemma 3.7. Let $\Lambda$ be a WR lattice in $\mathbb{R}^{2}$. A lattice $\Omega \subset \mathbb{R}^{2}$ is similar to $\Lambda$ if and only if $\Omega$ is also WR and $\theta(\Lambda)=\theta(\Omega)$.

Proof. First suppose that $\Lambda$ and $\Omega$ are similar. Let $\boldsymbol{x}_{1}, \boldsymbol{x}_{2}$ be the minimal basis for $\Lambda$. There exist a real constant $\alpha$ and a real orthogonal $2 \times 2$ matrix $U$ such that $\Omega=\alpha U \Lambda$. Let $\boldsymbol{y}_{1}, \boldsymbol{y}_{2}$ be a basis for $\Omega$ such that

$$
\left(\begin{array}{ll}
\boldsymbol{y}_{1} & \boldsymbol{y}_{2}
\end{array}\right)=\alpha U\left(\boldsymbol{x}_{1} \boldsymbol{x}_{2}\right) \text {. }
$$


Then $\left\|\boldsymbol{y}_{1}\right\|=\left\|\boldsymbol{y}_{2}\right\|$, and the angle between $\boldsymbol{y}_{1}$ and $\boldsymbol{y}_{2}$ is $\theta(\Lambda) \in[\pi / 3, \pi / 2]$. By Lemma 3.5 it follows that $\boldsymbol{y}_{1}, \boldsymbol{y}_{2}$ is a minimal basis for $\Omega$, and so $\Omega$ is WR and $\theta(\Omega)=\theta(\Lambda)$.

Next assume that $\Omega$ is WR and $\theta(\Omega)=\theta(\Lambda)$. Let $\lambda(\Lambda)$ and $\lambda(\Omega)$ be the respective values of successive minima of $\Lambda$ and $\Omega$. Let $\boldsymbol{x}_{1}, \boldsymbol{x}_{2}$ and $\boldsymbol{y}_{1}, \boldsymbol{y}_{2}$ be the minimal bases for $\Lambda$ and $\Omega$, respectively. Define

$$
z_{1}=\frac{\lambda(\Lambda)}{\lambda(\Omega)} \boldsymbol{y}_{1}, \quad z_{2}=\frac{\lambda(\Lambda)}{\lambda(\Omega)} \boldsymbol{y}_{2}
$$

Then $x_{1}, x_{2}$ and $z_{1}, z_{2}$ are pairs of points on the circle of radius $\lambda(\Lambda)$ centered at the origin in $\mathbb{R}^{2}$ with equal angles between them. Therefore, there exists a $2 \times 2$ real orthogonal matrix $U$ such that

$$
\left(y_{1} y_{2}\right)=\frac{\lambda(\Lambda)}{\lambda(\Omega)}\left(z_{1} z_{2}\right)=\frac{\lambda(\Lambda)}{\lambda(\Omega)} U\left(x_{1} x_{2}\right)
$$

and so $\Lambda$ and $\Omega$ are similar lattices. This completes the proof.

We are now ready to prove the main result.

Proof of Theorem 1.1. The density inequality (7) says that the largest lattice packing density in $\mathbb{R}^{2}$ is achieved by some WR lattice $\Lambda$, and (6) implies that

$$
\Delta(\Lambda)=\frac{\pi}{4 \sin \theta(\Lambda)}
$$

meaning that a smaller $\sin \theta(\Lambda)$ corresponds to a larger $\Delta(\Lambda)$. Lemma 3.2 implies that $\theta(\Lambda) \geq \pi / 3$, meaning that $\sin \theta(\Lambda) \geq \sqrt{3} / 2$. Notice that if $\Lambda$ is the hexagonal lattice

$$
\Lambda_{h}=\left(\begin{array}{ll}
1 & \frac{1}{2} \\
0 & \frac{\sqrt{3}}{2}
\end{array}\right) \mathbb{Z}^{2},
$$

then $\sin \theta(\Lambda)=\sqrt{3} / 2$, meaning that the angle between the basis vectors $(1,0)$ and $(1 / 2, \sqrt{3} / 2)$ is $\theta=\pi / 3$, and so by Lemma 3.5 this is a minimal basis and $\theta(\Lambda)=\pi / 3$. Hence the largest lattice packing density in $\mathbb{R}^{2}$ is achieved by the hexagonal lattice. This value now follows from (8).

Now suppose that for some lattice $\Lambda, \Delta(\Lambda)=\Delta\left(\Lambda_{h}\right)$, then by (7) and a short argument after it $\Lambda$ must be WR, and so

$$
\Delta(\Lambda)=\frac{\pi}{4 \sin \theta(\Lambda)}=\Delta\left(\Lambda_{h}\right)=\frac{\pi}{4 \sin \pi / 3}
$$

Then $\theta(\Lambda)=\pi / 3$, and so $\Lambda$ is similar to $\Lambda_{h}$ by Lemma 3.7. This completes the proof.

\section{Acknowledgement}

I would like to thank Sinai Robins for his helpful comments on the subject of this paper. 


\section{References}

[1] Banihashemi, A.H.; Khandani, A.K.: On the complexity of decoding lattices using the Korkin-Zolotarev reduced basis. IEEE Trans. Inform. Theory 44 (1998) 1, 162-171.

[2] Conway, J.H.; Sloane, N.J.A.: Sphere Packings, Lattices, and Groups. Springer-Verlag, Third edition, 1999.

[3] Fukshansky, L.: On similarity classes of well-rounded sublattices of $\mathbb{Z}^{2}$. To appear in: J. Number Theory; arXiv:0708.1596.

[4] Fukshansky, L.: On distribution of well-rounded sublattices of $\mathbb{Z}^{2}$. J. Number Theory 128 (2008) 8, 23592393.

[5] Fukshansky, L.; Robins, S.: Frobenius problem and the covering radius of a lattice. Discrete Comput. Geom. 37 (2007) 3, 471-483.

[6] Hsiang, W.Y.: Least Action Principle of Crystal Formation of Dense Packing Type and Kepler's Conjecture. World Scientific Publishing Company, 2002.

[7] Martinet, J.: Perfect Lattices in Euclidean Spaces. Springer-Verlag, 2003.

[8] McMullen, C.: Minkowski’s conjecture, well-rounded lattices and topological dimension. J. Amer. Math. Soc. 18 (2005) 3, 711-734.

[9] Pohst, M.: On the computation of lattice vectors of minimal length, successive minima and reduced bases with applications. Technical report.

[10] Rogers, C.A.: Packing and Covering. Cambridge University Press, 1964.

[11] Schuermann, A.: Computational geometry of positive definite quadratic forms. Polyhedral reduction theories, algorithms, and applications. University Lecture Series 48. American Mathematical Society, 2009.

[12] Szpiro, G.G.: Kepler's conjecture. How some of the greatest minds in history helped solve one of the oldest math problems in the world. John Wiley \& Sons, 2003.

Lenny Fukshansky

Department of Mathematics

Claremont McKenna College

850 Columbia Avenue

Claremont, CA 91711-6420, USA

e-mail: lenny@cmc.edu 